\title{
Atención durante el proceso de parto de adolescentes en la Cátedra y Servicio de Gineco-Obstetricia del Hospital de Clínicas, 2019
}

\section{Attention during the process of childbirth of adolescents in the Chair and Service of Gyneco-Obstetrics of the Hospital de Clínicas, 2019}

\author{
Sheyla María Acosta Soria ${ }^{a}$, Hugo Máximo Chamorro Mendieta ${ }^{a}$
}

\begin{abstract}
Resumen
Las formas utilizadas en la atención a adolescentes en proceso de parto es un tema del que existe escaza información, que requiere un amplio debate desde la perspectiva de derechos y su importancia es cada vez más relevante debido a la demanda de adolescentes que pasan por este proceso. El objetivo de esta investigación fue describir la atención recibida durante el proceso de parto de adolescentes en el servicio de Gineco-Obstetricia del Hospital de Clínicas de San Lorenzo, para lo cual se planteó caracterizar la atención que brindan los profesionales sanitarios a adolescentes durante el proceso del parto e identificar la vivencia de la adolescente en dicho proceso. El estudio fue de tipo exploratorio de carácter cualitativo siguiendo el método de manera descriptiva y analítica. El cometido fue mostrar un panorama general de la situación de las adolescentes embarazadas, como un primer acercamiento y por última describirlas. Los resultados coexistieron en las dimensiones en donde se describió la atención a las usuarias, las que expresaron satisfacción hacia cada procedimiento y servicios brindados por los profesionales, quienes les han brindado seguridad y empatía. Las respuestas a sus necesidades físicas y emocionales han quedado satisfechas. En conclusión, en cuanto a la atención recibida por las adolescentes que pasaron por el proceso del parto en el Hospital de Clínicas se enmarca dentro de los parámetros establecidos por la OMS de Parto Humanizado, limpio o digno.
\end{abstract}

Palabras clave: adolescente, atención, proceso del parto, parto humanizado.

\footnotetext{
Abstract

The forms used in the care of adolescents in the process of childbirth is a subject on which there is little information, which requires a
}

\author{
${ }^{a}$ Universidad Nacional \\ de Asunción, Facultad de \\ Ciencias Sociales, Paraguay. \\ Correspondencia a: \\ ssheyacos@hotmail.es \\ Recibido: \\ 31 de octubre de 2020 \\ Aceptado: \\ 19 de noviembre de 2020

\section{(c) (1)} \\ Artículo publicado en acceso \\ abierto bajo la Licencia \\ Creative Commons.

$$
\begin{aligned}
& \text { Cita: } \\
& \text { Acosta Soria, S. M., \& } \\
& \text { Chamorro Mendieta, H. M. } \\
& \text { (2020). Atención durante } \\
& \text { el proceso de parto de } \\
& \text { adolescentes en la Cátedra } \\
& \text { y Servicio de Gineco- } \\
& \text { Obstetricia del Hospital de } \\
& \text { Clínicas, 2019. Kera Yvoty: } \\
& \text { reflexiones sobre la cuestión } \\
& \text { social, 5, 44-57. }
\end{aligned}
$$


wide debate from the perspective of rights and its importance is increasingly relevant due to the demand of adolescents who go through this process. The objective of this research was to describe the care received during the adolescent birth process in the Gyneco-Obstetrics service of the Hospital de Clínicas de San Lorenzo, for which it was proposed to characterize the care that health professionals provide to adolescents during the process of childbirth and identify the experience of the adolescent in this process. The study was of a qualitative exploratory type, following the method in a descriptive and analytical way. The task was to show an overview of the situation of pregnant adolescents, as a first approach and finally describe them. The results coexisted in the dimensions where the care for the users was described, those who expressed satisfaction with each procedure and services provided by the professionals, who have provided them with security and empathy. The answers to your physical and emotional needs have been met. In conclusion, the care received by the adolescents who went through the process of childbirth at the Hospital de Clínicas is framed within the parameters established by the WHO of Humanized, clean or dignified delivery.

Keywords: adolescent, care, childbirth process, humanized delivery.

\section{Introducción}

Este trabajo fue realizado en base al trabajo final de grado presentado para optar por el título de licenciatura en Trabajo Social en la Facultad de Ciencias Sociales. El mismo se realizó en el marco del convenio de investigación denominado carrera de trabajo social - Proyecto de Investigación: PINV15-432 "Fortalecimiento de Atención de la Cátedra de Psiquiatría del Hospital de Clínica para detención, tratamiento y derivación judicial oportuna de casos de violencia hacia niños, niñas y adolescentes de la población consultante" seleccionado por el proyecto PROCIENCIA del Consejo Nacional de Ciencia y Tecnología (CONACYT).

El mismo buscó visibilizar el tipo de atención recibida por la adolescente en el proceso del parto en el ámbito hospitalario, situación compleja que se desarrolla en un escenario desconocido para la adolescente y en el que intervienen diversos actores.

Según informes de organizaciones internacionales (OPS, OMS, UNFPA, UNICEF, 2018) a nivel mundial cada año quedan embarazadas aproximadamente 16 millones de adolescentes, entre 15 a 19 años. Un estudio realizado en nuestro país en 2016 y actualizado en el 2018 expone que, según el Ministerio de Salud, entre 2010 y 2016, en promedio se produjeron unos 20.000 nacimientos anuales de hijos/as de madres adolescentes de entre 15 y 19 años. Es decir que 63 de los nacimientos que ocurrieron diariamente en el país en el mencionado periodo de tiempo son recién nacidos hijos/ as de adolescentes de entre 15 a 19 años. Lo expuesto implica que en este periodo de tiempo el $18,7 \%$ de todos los nacimientos registrados correspondió a nacidos vivos hijos/as de niñas y adolescentes de 10 a 19 años (CDE, 2018).

A nivel mundial, las complicaciones del embarazo y el parto son las principales causas de muerte en adolescentes de 15 a 19 años. El riesgo de muerte materna es menor en las madres en torno a los 20 años de edad, y máximo en las madres que dan a luz antes de los 15 años (OPS, OMS, UNFPA, UNICEF, 2018).

En ese contexto, en la búsqueda deuna atención acorde a la edad y características de la adolescente, la OMS lanzó en el 2014 los Estándares de Calidad para los Servicios de Salud para Adolescentes (ECSSA); documento suscripto por Paraguay.

La atención a la adolescente parturienta exige actitudes y comportamientos de las y los trabajadores de la salud, considerando la atención en la salud como un derecho humano, como la utilización de tecnologías que fortalezcan 
Acosta Soria, S. M., \& Chamorro Mendieta, H. M. Atención durante el proceso de parto de adolescentes en la Cátedra y Servicio de Gineco-Obstetricia del Hospital de Clínicas, 2019.

el vínculo entre profesionales de salud, usuarias, familias y comunidad (Silva et al., 2011).

Según la OMS (2018) a nivel mundial, a pesar de los importantes debates e investigaciones que se han desarrollado durante muchos años, el concepto de "normalidad" en el trabajo de parto y el parto, no es universal ni está estandarizado. En las últimas dos décadas, se ha producido un aumento considerable en la aplicación de diversas prácticas de trabajo de parto que permiten iniciar, acelerar, terminar, regular o vigilar el proceso fisiológico con el fin de mejorar los resultados tanto para las mujeres como para los bebés, pero dentro de esas diversas técnicas o métodos es importante identificar si esas prácticas incorporan un enfoque de derecho considerando a la población adolescente.

Es importante entender que esto sucede en un espacio institucional sanitario, en donde se ejercen relaciones de poder, principalmente desde el ámbito simbólico, recordando que por lo general el personal médico (y en general otro personal de la salud que se adhiere a este mismo discurso), es el que tiene el conocimiento. Desde este poder se ejerce dominio sobre los "cuerpos dóciles" al cual se refiere el filósofo y psicólogo francés Michel Foucault (1926-1984) en su libro Vigilar y Castigar (Foucault, 2002).

Desde esta perspectiva, la atención humanizada o la esencia del cuidado de la persona tendría poca relevancia, propiciando el ejercicio de diferentes formas de violencia invisibilizadas, normalizadas y en muchos casos naturalizadas por quienes las cometen y las sufren según el concepto de violencia cultural de Galtung. La violencia es entendida como un modo de relación que daña o perjudica física y/o psicológicamente, expone a las personas $y / o$ grupos en una situación de desventaja social frente a otros y vulnera los derechos humanos excluyendo o discriminando. Es transversal y afecta distintos ámbitos de la vida humana. Se conocen, por ejemplo; la violencia criminal o delictiva, de género, intrafamiliar, escolar, de Estado, entre otras; además se consideran actos y personas violentas o violentadas (Leyton \& Toledo, 2012).

Las experiencias empíricas en torno al embarazo y parto proporcionan conocimientos acerca de la atención que es presta a las mujeres gestantes; donde su decisión, opción o deseo además de su autonomía y libertad no siempre son tenidas en cuenta. Existen casos conocidos a través de los medios masivos de comunicación o las redes sociales, que dan cuenta de la existencia de víctimas de una atención hospitalaria; donde se vulneran los derechos sexuales y reproductivos.

Las primeras aproximaciones a bibliografías, trabajos finales de grado y estudios realizados sobre violencia obstétrica y esencia del cuidado y la percepción que reciben las adolescentes durante el parto dan cuenta de que es un tema escasamente abordado y mucho más en la franja etaria que alberga a la niñez y adolescencia, motivo por el cual en la incipiente fase exploratoria se obtuvo una acotada información

La realidad vivida por las adolescentes embarazadas está atravesada por una diversidad de variables; entre ellas su condición de mujer y de adolescente, categorías subvaloradas socioculturalmente, que la constituyen en objeto del paternalismo, control y tutela que las cosifican y discriminan dentro de una cultura patriarcal. La mayoría de las niñas adolescentes son obligadas a abandonar su formación académica a causa del embarazo y posteriores exigencias del cuidado afectan sus proyecciones económicas que le aseguren una vida digna con autonomía.

Esta realidad es invisibilizada dentro del sistema de salud, y más aún cuando se trata de la atención a adolescentes. Por esta razón es de gran importancia aumentar la producción científica sobre el parto humanizado o la situación opuesta; que presente características de la violencia obstétrica hacia todas las mujeres en general y las adolescentes en particular a partir de sus vivencias. 
En cuanto a las características del cuidado que reciben las adolescentes embarazadas en el ámbito hospitalario; tomando como indicador una atención integral, humanizada y multidisciplinaria que cuente con los principios expuestos en el Manual Clínico para el Manejo Integral de Adolescentes con Enfoque de Derechos del Ministerio de Salud Pública y Bienestar Social (2015), los resultados aportan elementos para la definición de políticas institucionales acordes a la situación y características de las mismas.

Así mismo ofrece una oportunidad a las adolescentes puérperas de expresar y dar a conocer sus vivencias respecto a la atención recibida en el proceso del parto. Además, posibilita el análisis o las acciones o actitudes del personal sanitario, de manera a identificar los tratos y procedimientos contrarios al enfoque de derecho, entendiéndola como expresión de violencia.

Los datos obtenidos favorecen la generación de procesos de reflexión para el mejoramiento del cuidado en estas situaciones y la calidad de los servicios de salud Gineco-obstétricos ofrecidos, brindando protección a las adolescentes en los servicios hospitalarios. Se aporta valiosa información para la concreción de políticas públicas enfocadas en la atención de las adolescentes embarazadas durante el proceso de parto y el desarrollo de acciones de prevención del maltrato y empoderamiento de las adolescentes una vivencia más positiva del parto.

Para enfatizar, este trabajo indagó sobre las vivencias de las adolescentes duranteel procesodeparto, reconociéndolas sujetas de derechos; considerando relevantes sus sentimientos, sensaciones y necesidades para conocer a profundidad la atención que recibió del profesional de la salud que la asistió.

El enfoque del estudio fue cualitativo, buscó describir, caracterizar e identificar la atención durante el proceso de parto de la adolescente desde la percepción de los actores involucrados. El estudio se llevó a cabo en la Cátedra y Servicio de GinecoObstetricia del Hospital de Clínicas de San Lorenzo.

A partir de lo expuesto, se planteó la siguiente pregunta principal de investigación: ¿Cuál es la atención recibida por las adolescentes durante el proceso del parto en la Cátedra y Servicio de GinecoObstetricia del Hospital de Clínicas de San Lorenzo - FCM/UNA- 2019?

El objetivo general fue describir la atención recibida durante el proceso de parto de adolescentes en el servicio de Gineco-Obstetricia del Hospital de Clínicas de San Lorenzo. En torno a esto se establecieron como objetivos específicos caracterizar la atención que brindan los profesionales sanitarios a adolescentes durante el proceso del parto e identificar la vivencia de la adolescente durante el proceso de parto.

\section{Características de la atención a las adolescentes durante el proceso de parto}

2.1. Descripción del trato recibido por las adolescentes de parte de los profesionales sanitarios

Las adolescentes puérperas perciben que fueron bien atendidas al ser recibidas en el Hospital de Clínicas. Los profesionales sanitarios entablan un trato amable durante la atención de los partos como lo plantea la OMS (2018), el cual se enmarca en los parámetros del parto humanizado, respetado, digno o limpio. Entre algunos aspectos de la buena atención señalan el ser llamadas por sus nombres, la utilización de diversas estrategias para hacerlas sentir cómodas y seguras en el proceso de parto. Esto demuestra respeto a la parturienta, reconocimiento como sujeta de derecho y una relación profesional-usuaria, contraria a la despersonalización de la atención, que busca superar la incapacidad de crear vínculos según lo planteado por la Comisión de Derechos Humanos del Distrito Federal (2016),

Haaa... oñatende poraiterei hikúai ko'ápe. Ha che aju hace diez días 
ha aju apyta ape albérguepe... laaa... upépe añema'érã hağuã Kuri (...) Oñatende poraiterei hikúai ko'ápe. E2

Princesa me decían, usaban palabras lindas. $E_{5}$

\subsection{Actuación del profesional sanitario respecto al procedimiento establecido para el proceso del parto}

Los profesionales sanitarios que asisten a las gestantes adoptan medidas que se anticipan a sus necesidades físicas y emocionales, entre ellas brindarles la posibilidad de darse un baño y de tomar agua, sentarse junto a ellas a conversar y ayudarlas a ponersu atención en otros temas para superar el nerviosismo y desenfocarlas del dolor, acariciarles la panza e indicarles como respirar. Que ellas sintiesen el respeto durante este proceso fue muy importante, porque implicaba una adecuada actuación profesional.

En cambio, otras adolescentes usuarias derivadas de otros centros de asistencia de gestión pública, ya en proceso de parto, percibieron que fueron tratadas de manera inadecuada, porque les practicaron procedimientos invasivos, utilizaron palabras que denotaban impaciencia cuando los profesionales sanitarios se dirigían a ellas.

No me dijeron nada, pero, en la revisión me metieron sin avisar la mano y me asuste, porque soy primeriza. Acá si, cada cosa que iban a hacerme, antes de hacerme me explicaban. E6

Estas experiencias dejan entrever que no fueron bien atendidas, habiendo recibido maltrato verbal y físico de parte de los profesionales que la asistieron. Actitudes que evidencian el insuficiente reconocimiento y valoración de las adolescentes como sujetas de derechos que producen exclusión, discriminación y subordinación. Todo esto las expone a un parto inseguro y en condiciones de vulnerabilidad como lo expresan Mulder et al. (2014).

Otro aspecto abordado es sobre las dudas que le surgían y el modo en que fueron respondidas por el profesional sanitario. Algunas puérperas expresaron que rápidamente fueron respondidas sus dudas, le explicaron todos los procedimientos que le harían, con información clara, precisa y veraz. En contrapartida otras respondieron que no tuvieron dudas o quedaban en silencio. Sin embargo, ante las experiencias de las adolescentes que no expresaron ni manifestaron nada se plantea la sumisión cultural al poder del saber médico o las limitaciones de la cultura sanitaria.

Entre los protagonistas del evento del nacimiento: la adolescente madre, el neonato y lo miembros de sus familias y su entorno, la comunicación es un elemento fundamental. Las entrevistadas entienden que ésta se dio de forma constante, relativamente inmediata y minuciosa de forma que sus acompañantes tengan información sobre los procedimientos que le eran practicados en el proceso de parto como lo menciona Pascualini y LLorens (2010). Por tanto, los profesionales no solo se ocupan de dar tranquilidad a la adolescente, sino también a su entorno familiar.

Sí, sí... sí o sí le decían cómo estaba yo para no dejarle preocupado ni nada. E1

Para la adolescente gestante es importante el acompañamiento de una persona de su entorno, mientras los personales sanitarios le ofrecen los cuidados médicos y técnicos. Investigaciones realizadas demuestran que este apoyo redunda en beneficios tanto al momento del parto como en el futuro para la madre y también para el neonato según la OMS (2014).

En las expresiones del profesional se observó la complejidad del embarazo de la adolescente, teniendo en cuenta algunos factores que intervienen como la edad y el origen del embarazo, que pudo ser 
resultante de una situación de abuso sexual. Y la paciente ingresa, se le indica en donde va a estar, se le acompaña, se le da cariño, se le trata de contenerle porque es muy jovencita y a veces no sabemos muchas veces como llegó a embarazarse, a veces son pacientes que fueron abusadas y se trata de tener cierto cuidado con ese aspecto, no se le pregunta luego mucho. $\mathrm{P}_{4}$

Sin embargo, existen ocasiones en que algunos profesionales sanitarios descuidan la implementación de las medidas necesarias para la atención de las adolescentes parturientas o puérperas.

Hay veces que lastimosamente los profesionales no la ven como una adolescente. La ven y le tratan como una adulta, lastimosamente; a veces. Ellas tienen su sala, ellas tienen que estar aisladas por ejemplo cuando están internadas. P6

Uno de los principales obstáculos para transformar las condiciones de los partos y los aspectos que deben ser priorizados para el logro de cambios positivos en los modelos de atención en Paraguay son aquellos asociados a las limitaciones en la infraestructura edilicia de los servicios de atención al proceso de parto, lo que resta posibilidades a la adolescente de que sea acompañada por un familiar o persona de su confianza, debido que las pacientes están juntas en una sala grande y las condiciones para que la adolescente reciba una atención aislada de las demás son mínimas, una cama se halla separada de la otra por una cortina. A más de que no pueden estar acompañadas por sus familiares también carecen de privacidad en una situación que significa una vivencia muy personal.

Ante esto los profesionales sanitarios implementan procedimientos alternativos que permitan a las adolescentes experimentar mayor libertad, favoreciendo que estén acompañadas de sus familiares o personas de su confianza con el constante monitoreo de sus condiciones biológicas durante los primeros momentos del trabajo de parto.

Lo que intentamos por eso es que tipo la paciente este afuera y paseándose que pase un proceso también de su trabajo de parto inicial recorriendo el hospital y después se le va controlando si o si cada media hora como para que pueda tener esa contención familiar un poquito más tiempo y después cuando tenga ya una dilatación un poco más avanzada es que entra ya a la sala de preparto (...) $\mathrm{P} 1$

Un elemento esencial para asegurar que el parto sea humanizado es la información recibida durante las consultas prenatales, como la formación de los profesionales para su actuación dentro de ese modelo o paradigma. Es necesario que los demás establecimientos de salud cuenten con un espacio como el Espacio Ñangareko del Hospital de Clínicas.

\subsection{La información, factor favorecedor para la vigencia del parto humanizado. Avances $y$ desafíos.}

En cuanto a las capacitaciones realizadas a través de charlas, talleres o consejería sobre el parto; algunas adolescentes mencionan que sí recibieron cierto tipo de charlas dentro de las consultas pre natales. Sin embargo, otra refirió que no recibió ningún tipo de información y una parte de las entrevistadas quedó en silencio.

El acceso a la información depende en gran medida del lugar donde se realizan las consultas prenatales. Es así que quienes acudieron al Servicio y Cátedra de GinecoObstetricia del Hospital de Clínicas han recibido, según sus expresiones; información oportuna durantesusconsultas prenatales en la Clínica Fundación Juan Rasmuss Echecoper "Ñangareko".

Otrasqueacudieronaestablecimientos sanitarios de gestión pública cercanos a sus domicilios no tuvieron el mismo tratamiento mencionado con anterioridad. 
Acosta Soria, S. M., \& Chamorro Mendieta, H. M. Atención durante el proceso de parto de adolescentes en la Cátedra y Servicio de Gineco-Obstetricia del Hospital de Clínicas, 2019.

La información recibida por las adolescentes gestantes en las consultas prenatales les brinda la posibilidad de desarrollar un proceso de parto exitoso, satisfactorio y seguro. Les proporciona, además; de valor, la posibilidad de conocer y manejar técnicas de respiración y recibir indicaciones para afrontar la cesárea.

El desconocimiento indudablemente limita la capacidad de reconocer que la asistencia sanitaria hospitalaria durante el proceso del parto debe ser realizada bajo ciertas características y en determinadas condiciones establecidas y contempladas en la legislación nacional e internacional, que buscan garantizar la salud y el bienestar de las personas involucradas en el evento del nacimiento.

Todas las adolescentes refirieron que contaron con información suficiente para afrontar el proceso del parto. Además, la experiencia exitosa, satisfactoria y positiva del alumbramiento, según lo expresado por las usuarias del servicio era lo más importante.

Los profesionales de la salud expresan que las adolescentes gestantes reciben información acerca del proceso de parto, ya que se reconoce la importancia de que la usuaria cuente con información referente al evento que debe afrontar y los procedimientos que se les va a practicar. La información también es proporcionada a los familiares o personas que las acompañan. Estas prácticas y/o intervenciones ayudan a disminuir el miedo y la ansiedad de las adolescentes y sus familias.

Para quitarle un poquito lo que es el miedo y la ansiedad de eso, porque por más de que sean adolescentes, hasta las adultas a veces tenemos miedo (...) las adolescentes con más razón, están todas por igual asustadas quieren saber qué es, ¿cómo es, ¿qué me van a hacer? $\mathrm{P} 1$

Lo expuesto por los profesionales de la salud coincide con Barbieri, Soares, Ferrari, Demitto y Tacla (2013); mencionados por Centenaro et al. (2015); que sostienen que aquellas gestantes que fueron atendidas en el prenatal pasaban el proceso de parto con menos angustia y nerviosismo por las informaciones y consejos de preparación, conocer lo que les sucedería y cuál sería la mejor forma de atravesarla. Los autores referenciados investigaron sobre la correspondencia de las expectativas con las experiencias del parto de las adolescentes, y hallaron que la falta de información fue asociada a una mayor lentitud y dolor en el proceso de alumbramiento. Estas experiencias podían ser más armoniosas si las adolescentes hubiesen sido mejor informadas, los resultados positivos se relacionan con la mejora en la información al evaluar la experiencia del parto de la adolescente según lo expuesto por Morais et al. (2012); Schaffer et al. citados por Centenaro Levandowski et al. (2015).

\section{Vivencias de las adolescentes durante el proceso de parto}

3.1. Percepciones de las adolescentes puérperas sobre el trato durante el proceso del parto

Las adolescentes usuarias del servicio Gineco-obstétrico relataron que experimentaron nerviosismo y/o miedo durante la atención del proceso del parto, principalmente al inicio del trabajo de parto por la ansiedad que les producía el desconocimiento de lo que iba a pasar con ellas y al observar los equipamientos de la sala. A pesar de que las adolescentes contaban con información sobre cómo sería el parto, sintieron temor ante lo desconocido. Al trascurrir el tiempo dentro de la sala de parto fueron adquiriendo confianza en el entorno donde se encontraban y gradualmente iban sintiéndose mejor.

Ha upépe che nerviosa'íkuri... poo añe sentí porãã... E2

Sentía nervios, y la sala era cálida. $\mathrm{E}_{4}$

En uno de los casos la adolescente tuvo un parto normal y otras dos expresaron haber tenido su parto por cesárea. En las manifestaciones verbales y gestuales de la adolescente que tuvo un parto normal 
se percibe un sentimiento de satisfacción por haber podido dar a luz naturalmente, aunque en un primer momento los profesionales no lo creían viable.

Decía que no iba a poder tener un parto normal iba a tener por cesárea... Pero pude E1

Las adolescentes que expresaron haber tenido un parto por cesárea, refirieron que fue por problemas cardiovasculares en un caso y la otra por el tamaño del bebé. El conocimiento acabado de los motivos y las implicancias del tipo de parto al que debían someterse evidencian el empoderamiento de las adolescentes ante el evento.

\subsection{Sensaciones de las adolescentes sobre los procedimientos de los profesionales sanitarios en relación a sus necesidades emocionales durante el parto}

Las experiencias vividas en el contexto hospitalario se instalan en cada individuo e indiscutiblemente definen y determinan su percepción y sensación frente a las acciones de sus cuidadores (Hamui et al. 2013).

Dentro de la sala de parto se podrían dar estímulos negativos como positivos, como la empatía y el uso de palabras contenedoras, en un tono suave. Es lo que describen las adolescentes sobre su experiencia durante el proceso del parto al recordar con emoción que para tranquilizarla le hicieron escuchar una música de su agrado, le acompañaban para realizar algunos ejercicios.

(...) Que cuando me sentía nerviosa, sentía tanto dolor, vino la doctora y me dijo que si quería escuchar música y vino me puso la música que yo quería y me hablaba así y me traía en los ejercicios para hacer... todo eso... me ayuda. E1

La presencia con calidad y calidez del profesional sanitario que acompaña el momento de alumbramiento brindando la información oportuna de los procedimientos que se iban realizando generaba en las adolescentes puérperas confianza y disipaba los miedos. El primer contacto y encuentro con sus hijos daba una fuerza motivadora a las madres para afrontar las peripecias del alumbramiento.

\subsection{Contención y acompañamiento}

El respeto a la intimidad de la adolescente parturienta es un componente esencial a ser tenido en cuenta en el marco de una atención integral durante el proceso de parto. Todas las adolescentes consultadas afirmaron sentirse respetadas en su intimidad por parte de los profesionales que le atendieron en el parto.

Una adolescente comentó que en otro establecimiento sanitario; donde concurrió previamente antes de ingresar al Hospital de Clínicas, experimentó un procedimiento del personal sanitario donde se sintió invadida en su intimidad, sumado a una situación de maltrato.

La cantidad de profesionales que intervienen dentro de la sala durante el parto puede generar sentimientos de incomodidad, o pasar como una situación irrelevante. Lo importante para las adolescentes madres fue contar con la constante asistencia de un profesional sanitario a su lado, acompañándolas muy de cerca en todo el proceso y comentándoles qué tipo de intervenciones se les practicarían.

Siendo la Cátedra y Servicio de Gineco-Obstetricia del Hospital de Clínicas de San Lorenzo un Hospital Escuela, la cantidad de profesionales dentro de la sala de parto sería mayor a lo habitual de otros hospitales, normalmente según los profesionales oscilan entre 6 a 8 profesionales en la sala de parto.

\subsection{Percepción de la atención}

Algunas de las adolescentes comentaron que no tuvieron ningún tipo de dudas durante el proceso del parto y una quedó en silencio. Señalaron que no tomaron la decisión de cómo sería su parto, dejaron que los médicos decidieran, dada la edad que tienen difícilmente podrían 
Acosta Soria, S. M., \& Chamorro Mendieta, H. M. Atención durante el proceso de parto de adolescentes en la Cátedra y Servicio de Gineco-Obstetricia del Hospital de Clínicas, 2019.

expresar sus deseos y decisiones, porque solo se aborda información con ellas pero no se tiene el suficiente tiempo para abordar con ellas su autonomía. Una de las adolescentes consultantes en el consultorio de Ñangareko mencionó que ella participó de la decisión de cómo sería su parto. Otra expresó haber dado a luz por parto normal a pesar de que en un primer momento del trabajo de parto los profesionales lo consideraban inviable.

Los profesionales sanitarios conversan con las adolescentes, no la dejan sola y le dan palabras de aliento y en momento de algún desborde de emociones solicitan a algún familiar que ingrese, de manera a hacerle sentir más acompañada (en la ante sala al quirófano). En situaciones que ameritan recurren a la psicóloga, pero también hasta la sala de pre parto.

Los profesionales sanitarios entienden quelasadolescentes consultantes en el consultorio de Ñangareko pasan por un mejor parto. Contar con información completa favorece que el parto sea más llevadero para las adolescentes.

Las experiencias desagradables, que relatan durante el proceso de parto son los dolores y la atención recibida en otros hospitales. Otras adolescentes puérperas no dieron ninguna respuesta a este planteamiento.

Los datos relevados coinciden con las prácticas en la asistencia del parto normal establecidas por la OMS, categorizadas como prácticas útiles y benéficas para el trabajo de parto y parto entre las que se mencionan que la mujer recibe el máximo de informaciones y explicaciones; libertad de posición y movimiento durante el trabajo de parto; técnicas no-invasivas y nofarmacológicas de alivio del dolor durante el trabajo de parto, como masaje y técnicas de relajación; y contacto precoz piel a piel entre madre y bebé y el apoyo al inicio del amamantamiento materno en la primera hora del posparto (Andrade et al., 2008).

\subsection{Sentimientos}

Luego del proceso de parto las adolescentes gestantes señalan que tienen sentimientos de tranquilidad, satisfacción por haber recibido una buena atención, alivio luego de los fuertes dolores y felicidad al tener contacto con su bebé.

Sentí toda una tranquilidad, se me fue dolor de mí y ya... yaaa me sentí aliviada súper tranquila ahí. E1

Las expresiones de las adolescentes puérperas permiten considerar que sus sentimientos al momento de ser atendidas durante el proceso de parto están estrechamente relacionados al trato que recibieron de parte de los profesionales sanitarios.

Algunas adolescentes puérperas enunciaron que la experiencia fue muy rápida por lo que no tenía nada que mencionar al respecto. Otras adolescentes madres mencionaron que el momento en que mantuvieron contacto con sus bebés les hizo sentir mucha tranquilidad y felicidad. Y finalmente algunas calificaron de bueno o muy bueno sus sentimientos en la experiencia de atención de su parto. Una de las adolescentes puérperas no brindó ningún dato del tema señalado, limitándose a decir no sé.

\subsection{Consideraciones sobre la intervención desde el trabajo social en la atención a las gestantes}

En este apartado se analiza la práctica del profesional de trabajo social desde la perspectiva crítica (Faleiros, 1987, 2007, Alayon, 2007, Netto, 1975, citados en Faleiros, 2011). Aunque este trabajo se ha enfocado en la atención del proceso de parto, se tiene presente que éste es solo uno de los momentos de todo el proceso reproductivo que abarca la salud sexual, el embarazo, parto y post parto (Belli, 2013). Y cada uno de estos momentos son objetos de la intervención de diferentes profesionales incluido el trabajador social.

El profesional de trabajo social brinda asistencia en los servicios ginecológicos de las instituciones hospitalarias a toda mujer, 
adolescente o adulta que demanda atención en relación a su salud sexual en general; entre ellas se encuentran las gestantes. El embarazo es un evento que requiere de una intervención donde se evalúe, analice y reconstruya las condiciones de vida de la usuaria directamente y de todo su entorno. Para lo cual se deben identificar sus determinaciones y mediaciones; superando la visión inmediata, fragmentada y estática de los 'problemas sociales' (Millardi, 2013). Considerando lo expuesto la/el profesional entrevistado ha comentado que son frecuentes las intervenciones con adolescentes que muchas veces atraviesan por embarazos consecutivos. Sin embargo, resulta llamativa la normalidad en el comentario respecto a las bajas de las edades de los embarazos de las adolescentes, aun cuando no se debe perder la indignación sobre dichas situaciones.

Muchas veces tenemos una adolescente de 16 años y ya es su segundo embarazo (...), nosotros ya no nos espantamos cuándo te viene una de 13 y 14 años. P6

La falta de acompañamiento a las gestantesolasparejasmuymayoresestambién otra situación problemática gestionada, se evidencia poco cuestionamiento de ese tipo de relaciones, donde es probable existan otros factores que deben ser analizados, como posibles hechos de abuso sexual.

El embarazo y la maternidad en la adolescencia es considerada una problemática con diversas fuentes explicativas, propias de una realidad compleja que debe ser abordada más allá de una lógica sanitaria, en torno a ella existen varios cuestionamientos respecto a la suficiencia de las alternativas existentes, las categorías analíticas que ayudarían a entender el fenómeno, los factores que constituyen la realidad de las adolescentes, los enfoques que deben ser analizados y el papel de las políticas sociales, actualmente focalizadas y residuales (Escobar, 2011). Las opiniones profesionales dejan traslucir estereotipos propios de una sociedad que culpabiliza y responsabiliza de la situación a las adolescentes.

La mamá vino llorando, pidiéndonos auxiiilio, que volvamos a intervenir. $Y$ con sus trece añitos ya tiene una mentalidad, no es de una de trece años (...) P6

Las situaciones de violencia doméstica o intrafamiliary sus implicancias son frecuentes entre las usuarias del servicio. Estas situaciones derivan en casos judicializados en los que se presentan obstáculos como falta de seguimiento a los casos y no concurrencia de los funcionarios de las instancias correspondientes.

Muchos casos abordados son mediatizados, y se percibe que solo cuando son conocidos a través de los medios de comunicación o las redes sociales son debidamente atendidos.

Las madres adolescentes sobrellevan situaciones complicadas que trascienden su situación, que no es exclusiva de los sectores más pobres, pero se da mayoritariamente en esta; retroalimentándose un ciclo muy perverso de vulnerabilidad social y de exclusión, relacionado con el abandono del sistema educativo, aunque muchas veces esta no es su principal causa.

A todas estas situaciones se suman las condiciones propias de la institución empleadora del profesional que no pueden ser obviadas considerando que la práctica profesional es un trabajo y está inserto en el proceso del trabajo (Iamamoto, 1998), y por ende condicionan la labor de la o el trabajadora social.

\subsection{La identidad del profesional de Trabajo Social en la división socio- técnica del trabajo}

Se planteó a las profesionales como entiende la función del trabajo social dentro del contexto de la atención a las mujeres embarazadas, sean adolescentes o adultas. Según sus expresiones, se actúa ante los riesgos en cuanto a la falta de recursos económicos necesarios para el cuidado de su salud. Atribuye también 
Acosta Soria, S. M., \& Chamorro Mendieta, H. M. Atención durante el proceso de parto de adolescentes en la Cátedra y Servicio de Gineco-Obstetricia del Hospital de Clínicas, 2019.

como objeto de la intervención la asistencia de aquellas gestantes que no cuentan con el acompañamiento de sus familiares o personas de confianza o ante casos sospechosos o confirmados de violencia.

Lo expuesto nos remite al trabajo social como la profesión que orienta su intervención a la atención de necesidades de personas, familias, gruposy poblaciones que tienen dificultades para la reproducción de su existencia, promoviendo su vinculación con instituciones públicas y privadas que disponen o pueden crear satisfactores (Aquín et al., 1995).

\section{Resultados y/o conclusiones}

La investigación da cuenta que toda atención a la gestante durante el proceso de parto está determinada por múltiples factores, haciendo referencia al objetivo de caracterizar la atención que brindan los profesionales sanitarios a las adolescentes durante el proceso del parto.

Además de las embarazadas, sus bebés y el personal sanitario que la asistía en el momento del alumbramiento, se involucraban también de manera indirecta los familiares y/o personas del entorno de la parturienta. La descripción del trato recibido por las adolescentes de parte de los profesionales sanitarios según sus percepciones estuvo basada en la satisfacción y trato amable, enmarcados en los estándares establecidos para el parto humanizado, respetado, digno o limpio. La identidad de cada adolescente parturienta, como su particular proceso fue respetado, fue reconocida.

La actuación del profesional sanitario respecto al procedimiento establecido para el proceso del parto ha sido diferenciada según el establecimiento sanitario al que acudieron. El trato recibido en los hospitales del sistema de salud pública fue distinto al brindado en el Hospital de Clínicas. Adolescentes que fueron trasladadas de otros hospitales, luego de haberse iniciado el trabajo de parto, fueron víctimas de violencia obstétrica según relatan en sus experiencias.
Dentro del establecimiento hospitalario durante la asistencia del proceso de parto se establecía una relación interpersonal entre la adolescente y el profesional sanitario, que desarrollaba con ellas una actitud empática.

Se visibiliza que aportando información dentro del desarrollo de la atención prenatal posibilitaría que las adolescentes reconozcan que la asistencia sanitaria hospitalaria durante el proceso del parto debe ser realizada bajo ciertas características y en determinadas condiciones establecidas. En cuanto a ello, todavía es insuficiente la información que reciben las mismas y el desconocimiento necesaria e indudablemente limita la capacidad de reconocerla.

En el proceso del parto cada caso requiere un trato y abordaje diferenciado de acuerdo a las necesidades físicas y emocionales de la parturienta, quien es la prioridad. El proceder médico debe propiciar el empoderamiento de las adolescentes ante el evento. La satisfacción de la atención recibida se percibió en el nacimiento exitoso del bebé, ocurrido sin demoras ni complicaciones, y el primer contacto y encuentro de las madres con sus hijos. Todo esto les concedía una fuerza motivadora para afrontar las vicisitudes del alumbramiento.

Respecto a la satisfacción de sus necesidades emocionales durante el parto, el trabajo advierte que se vivencia la calidez y calidad del profesional sanitario que acompañaba el momento del alumbramiento brindando la información oportuna de los procedimientos, lo que generaba confianza y disipaba los miedos al parto de las adolescentes puérperas.

La contención y acompañamiento están sujetos a la cantidad y calidad de los profesionales. Ante la cantidad de profesionales que intervinieron dentro de la sala durante el parto, lo que podía generar incomodidad para las adolescentes fue un factor irrelevante. Lo importante fue contar con la constante asistencia de un profesional sanitario a su lado en todo 
el proceso e informándoles sobre el tipo de intervenciones que se les practicaría.

El acompañamiento de una persona de su entorno estaba imposibilitado por la falta de infraestructura y materiales hospitalarios. Esto se debió a que todas las usuarias del servicio se encontraban juntas en una gran sala. Con lo cual éste es uno de los principales obstáculos para transformar las condiciones de los partos y es un aspecto que debe ser priorizado para el logro de cambios positivos en los modelos de atención en Paraguay.

Otro gran desafío es la superación de una suerte de confrontación generacional de profesionales sanitarios en cuanto a la hermenéutica de los modelos o paradigmas de la atención del proceso de parto que tensionan la práctica profesional hospitalaria y su concepción epistemológica. Una perspectiva tradicional médico hegemónico; y la otra mirada emergente en la que se busca una atención al parto con los nuevos estándares basados en los Derechos Humanos; acorde a los requerimientos establecidos nacional e internacionalmente para un parto humanizado, limpio o digno opuesto a las prácticas sanitarias consideradas en la legislación pertinente como violencia obstétrica.

Las prácticas en la asistencia del parto humanizado, respetado, digno o limpio son una realidad en las experiencias de las usuarias entrevistadas de la Cátedra y Servicio de Gineco-Obstetricia del Hospital de Clínicas, 2019, respondiendo al objetivo general de esta investigación sobre la atención recibida por las adolescentes durante el proceso del parto.

Porlo expuesto seentiendequea pesar de la existencia de una política de salud que establece y garantiza condiciones, medidas infraestructurales, de provisión de estudios médicos, medicamentos como también de prácticas del profesional sanitario en relación al tema abordado aún existen limitaciones y obstáculos que necesitan ser superados.

Durante el relevamiento de los datos las adolescentes entrevistadas han quedado en silencio en varios momentos ante ciertos temas que se planteaban, además se obtuvieron respuestas muy acotadas en varios casos. Ante estas conductas podría entenderse que el estado de puerperio en que se encontraban las adolescentes madres influía en sus capacidades de respuesta. Por otro lado, se plantea el bajo nivel de cultura sanitaria de las adolescentes usuarias del servicio.

Cabe señalarque los resultados de esta investigación no pueden ser generalizados por tratarse de un estudio cualitativo, el aporte que brinda guarda relación a la aproximación que hace a la problemática planteada inicialmente. Siendo la atención de la adolescente durante el proceso de parto un tema muy escasamente abordado, se plantea la necesidad y relevancia de llevar a cabo más estudios sobre el mismo.

\section{Recomendaciones}

Es imperioso que los estamentos de profesionales sanitarios refuercen las medidas ya implementadas referentes a lo conocido como atención integral y/o humanizada del parto. Con ello, se podría instalar una mirada crítica y oportuna a la realidad y condiciones socioeconómicas, sociales y culturales en torno a las adolescentes madres y la manera en que es abordada desde las políticas públicas. Desde el Trabajo Social se debería avanzar en ampliar instancias de intervención en las condiciones mencionadas de las gestantes en general y de las adolescentes en particular.

Por último, es necesario impulsar investigaciones sobre el proceso del parto de las adolescentes para analizar esta problemática de manera integral.

\section{Financiación}

Este proyecto es financiado por el CONACYT a través del programa PROCIENCIA, con recursos del Fondo para la Excelencia de la Educación e Investigación - FEEI del FONACIDE. 
Acosta Soria, S. M., \& Chamorro Mendieta, H. M. Atención durante el proceso de parto de adolescentes en la Cátedra y Servicio de Gineco-Obstetricia del Hospital de Clínicas, 2019.

\section{Referencias}

Andrade, A., Vasconcelos, A., \& Ferreira, E. (2008). Atención humanizada del parto de adolescentes: ¿norma, deseo o realidad? Obtenido de https:// scielo.conicyt.cl/pdf/rchog/v73n3/ arto8.pdf

Aquín, N., Custo, E., Torres, E. (2012). El Problema de la Autonomía en el Trabajo Social. Revista de Trabajo Social - FCH - UNCPBA. de https:// revistaplazapublica.files.wordpress. com/2014/06/7-19.pdf

Belli, L. F. (2013). La violencia obstétrica: otra forma de violación a los derechos humanos. Revista Redbioética/ UNESCO, 4(7), 28.

Centenaro Levandowski, D., Helena Marin, A., Pereira, C. S., \& Golembiewski Mendes, M. (2015). ¡Llegó la hora! Expectativas y experiencias de parto deadolescentes embarazadas. Revista de Psicología (PUCP), 33(2), 333361. http://www.scielo.org.pe/scielo. php?script $=$ sci_arttext $\&$ pid $=$ So254$92472015000200004 \& \operatorname{lng}=$ es\&tlng= es.

Centro de Documentación y Estudio, CDE. (2018). Embarazo y Maternidad de niñas en Paraguay. CDE. www.cde. org.py

Comisión de Derechos Humanos del Distrito Federal. (2016). Embarazo en Adolescentes y el Ejercicio de los Derechos Sexuales y Reproductivos en la Ciudad de México. https:// piensadh.cdhdf.org. $m x /$ images/ $\mathrm{public}$ a c i on es / I n form e mujeres/2015_Informe_mujeres.pdf

Escobar, M. (2011). Madres adolescentes, un abordaje desde la actual cuestión social (Tesis Licenciatura en Trabajo Social). TTS_EscobarMauricio\%20 (3).pdf

Faleiros, V. (2011). Desafíos del trabajo social frente a las desigualdades. Emancipação, Ponta Grossa, 11(1), 117128.

Foucault, M. (2002). Vigilar y Castigar. Nacimiento de la prisión. Siglo XXI Editores.

Hamui, L., Fuentes, R., Aguirre, R., \& Ramírez de la Roche, O. (2013). Expectativas y experiencias de los usuarios del Sistema de Salud en México; un estudio de satisfacción con la atención médica. http:// www.facmed.unam.mx/bmnd/ Satisfaccion AtencionMedica.pdf

Iamamoto, M. V. (1998). El Servicio Social en la Contemporaneidad: Trabajo y Formación Profesional. https://www. academia.edu/19323934/Iamamoto_ Marilda_El_Servicio_Social_en_la_ Contemporaneidad

Ministerio de Salud Pública y Bienestar Social, MSPyBS. (2015). Manual Clínico para el Manejo Integral de Adolescentes con Enfoque de Derechos. MSPyBS.

Mulder, S.; Castro, M., \& Fernández, C. (2014). Vivencias y relatos sobre el embarazo en adolescentes: Una aproximación a los factores culturales, sociales y emocionales a partir de un estudio en seis países de la región. Informe final. Ciudad del Saber. Plan Internacional- UNICEF.

OPS, OMS, UNFPA, UNICEF. (2018). Acelerar el progreso hacia la reducción del embarazo en la adolescencia en América Latina y el Caribe. Washington, D.C., EE. UU. https:// lac.unfpa.org/sites/default/files/ pub-pdf/ESP-EMBARAZO-ADOLES14febrero\%2oFINAL_5.PDF

Organización Mundial de la Salud, OMS. (2014). Prevención y erradicación de la falta de respeto y el maltrato durante la atención. http://www. who.int/reproductivehealth/topics/ maternal_perinatal/statementchildbirth/es/

Organización Mundial de la Salud, OMS. (2018). Recomendaciones de la OMS Para los cuidados durante el parto, para una experiencia de parto positiva Transformar la atención a mujeres y neonatos para mejorar su salud y bienestar. https://apps.who.int/iris/ bitstream/handle/10665/272

Pascualini, D., \& LLorens, A. (2010). Salud y Bienestar de los Adolescentes: Una Mirada integral. OPS/ OMS. http://www.corajic.org/ cites/www.corajic.org/files/ saludbienestaradolescente $\% 20$ Diana\%20compiladores.pdf 
Acosta Soria, S. M., \& Chamorro Mendieta, H. M. Atención durante el proceso de parto de adolescentes en la Cátedra y Servicio de Gineco-Obstetricia del Hospital de Clínicas, 2019..

\section{Sobre los Autores}

Sheyla María Acosta Soria

Licenciada en Trabajo Social por la Facultad de Ciencias Sociales de la Universidad Nacional de Asunción.

\section{Hugo Máximo Chamorro Mendieta}

Licenciado en Trabajo Social por la Facultad de Ciencias Sociales de la Universidad Nacional de Asunción. 